\title{
A MULTICENTER STUDY TREATMENT ADHERENCE OF HYPERTENSION FOCUSED ON PRIMARY HEALTH CARE IN INDONESIA
}

\author{
ENDANG SULISTIYOWATININGSIH, MUTIARA HERAWATI
}

Pharmacy Department, Islamic University of Indonesia, Indonesia

Email: endang.sn@uii.ac.id

Received: 05 Feb 2017 Revised and Accepted: 18 Apr 2017

\section{ABSTRACT}

Objective: Hypertension today has a remained a focus in developing countries. This study aim was to determine the treatment adherence measured by the 8-item Morisky Medication Adherence Scale (MMAS-8) in hypertensive outpatients.

Methods: A cross-sectional study was carried out with hypertensive patients older than $18 \mathrm{y}$, treated at eight of the primary health care in Special Region of Yogyakarta through interviews, between January and April 2016. Adherence was determined by MMAS-8 version translated for this study.

Results: The patients consider low adherence (score<6), moderate adherence (score 6-7) and high adherence when they had a score equal to 8 at the MMAS-8. The prevalence of adherence among the 233 patients in primary health care showed the majority was lower adherence (57.90\%), the moderate adherence (30.25\%) and the high adherence (11.82\%). The average adherence value according to the MMAS-8 was 5.2 ( \pm 1.7$)$.

Conclusion: Non-adherence treatments of hypertensive outpatients in primary health care was highest through application of MMAS-8

Keywords: Adherence, MMAS, Hypertension, Primary health care

(c) 2017 The Authors. Published by Innovare Academic Sciences Pvt Ltd. This is an open access article under the CC BY license (http://creativecommons.org/licenses/by/4.0/) DOI: http://dx.doi.org/10.22159/ajpcr.2017v10s3.21356

\section{INTRODUCTION}

Hypertension remains a considerable issue in every corner of the world. The increasing prevalence of hypertension in most countries is a special challenge against the risk of complication incidence, particularly in such vital organs as heart and kidneys. The prevalence of cardiovascular disorder will tend to increase each year if the countermeasures against hypertension are not best performed, A report from the Joint National Committee on Hypertension revealed that the number of hypertensive patients in the world has reached 1 million [1]. The incidence of hypertension today has remained a focus in developing countries, reaching $37.7 \%$, and is estimated to rise by three-quarters of the whole hypertensive population worldwide by the year 2025 [2]. Several studies in different countries indicated that antihypertensive therapy has proved to be clinically effective for countermeasures in hypertension.

However, many patients do not adhere to the recommended therapy; as a result, cases of hypertensive therapy failure frequently occur. It is proven by the level of blood pressure that has yet to meet the expected target. Based on a study by WHO, the main issue related to therapy management for patients with the chronic disease worldwide is their adherence to the given medication therapy. This incidence has reached a significant point up to $50 \%$ and is even likely to rise further in developing countries [3]. Uncontrolled hypertension can escalate the IHD risk by 3-4 times and become the risk factor of cardiovascular incidence by 2-3 times [4]. Even recent case control studies showed an increase in the risk of stroke incidence in poorlytreated hypertensive patients [5].

Since the enactment of universal coverage in Indonesia in 2014, primary health care centers play a major role as the center for primary service of public health in Indonesia. Sound, appropriate health care in a primary healthcare center is essential as the basis for establishing public health financing. In this context, the research was conducted on a disease with the highest prevalence in Indonesia, which is hypertension, particularly in a primary healthcare setting, by focusing on adherence to therapy. The method used to measure therapy adherence was MMAS-8 as it has a greater ability [6].

\section{MATERIALS AND METHODS}

\section{Ethical approval}

This study received approval from the Ethics Committee of the Faculty of Medicine, Islamic University of Indonesia and Health Service of Special Region Yogyakarta for 8 primary health care. Written consent was also obtained from the patients prior to the commencement of the study.

\section{Study setting, design and period}

Indonesia is an archipelago country which consists of 34 province. Yogyakarta is the capital of Yogyakarta Special Region province. A prospective cross-sectional study was carried out in 8 primary health care in Special Region of Yogyakarta spread across the city.

We investigated the adherence of antihypertensive treatment using the eight-item Morisky Medication Adherence Scale (MMAS). The MMAS-8 an update with greater sensitivity and the most commonly used self-reporting method to determine adherence, contains eight questions with closed dichotomous (yes/no) answer, designed to prevent the bias of positive responses from patients questions asked by health professionals, by reversing the responses related to the interviewee's adherence behavior [7].

MMAS consists of 8 items with a dichotomous response (yes/no) for items 1-7 and a 5 point Likert response for the last item. The total score ranges from 0 to 8 with a higher total score indicating higher medication adherence. The total score for each patient is the summation of the scores in each item. The degree of adherence was determined according to the score resulting from the sum of all the correct answers: high adherence (eight points), moderate adherence ( 6 to $<8$ points) and low adherence ( $<6$ points). In this study, patients were considered adherent when they had a score equal to eight in the MMAS-8. The MMAS showed a good predictive power and was 
significantly associated with drug pharmacy refill as it showed a $75 \%$ concordance [8].

\section{Study population}

We selected out patients with confirmed diagnosis of hypertension who were treated at primary health care, aged 18 or older and who used antihypertensive medications. Patients with secondary hypertension and compelling indication (Diabetes mellitus, heart disease, hyperlipidemia, stroke, and renal failure) confirmed by medical records were excluded. This exclusion criterion was aimed to eliminate the interference of the cost of drugs, polypharmacy, effectivity of the antihypertensive treatment as the major predictive factors of nonadherence.

\section{RESULTS AND DISCUSSION}

A total of 233 patients were eligible for participation in the study and eight of whom had been excluded from participating in the study (refusal rate $=3,4 \%$ ). In this context, the simplicity of the 8 -item
Morisky Medication Adherence Scale (MMAS-8) as a self-reported measure could make it a very practical approach to assess adherence to an antihypertensive treatment regimen. Although these methods are subject to bias, as the overestimation of adherence, the constant improvement of these instruments and their validation studies in different populations have increased their adoption in clinical practice [6].

More than $40 \%$ patients were not forgetting to take antihypertensive medications although they leave the house/travel and having no days on which medication was not taken during the previous $2 \mathrm{w}$ $(56.22 \%)$. In contrast, more than $70 \%$ of respondents reported that they had taken their hypertension medications on the previous day and that they had not decided to stop or reduce their hypertension medications when they felt worse but more than $49 \%$ decided to stop their hypertension medications when they felt better. In addition, most respondents never (75.97\%) or sometimes $(12.02 \%)$ had difficulty remembering their medications. The distributions of responses to each item on the 8-item MMAS are shown in table 1.

Table 1: Distribution of responses to items on 8-item MMAS-8

\begin{tabular}{|c|c|c|c|c|c|}
\hline \multicolumn{3}{|l|}{ Item 1-7 } & Yes (\%) & \multicolumn{2}{|c|}{ No (\%) } \\
\hline \multicolumn{3}{|c|}{ Do you sometimes forget to take your blood pressure medication? } & 50.21 & \multicolumn{2}{|c|}{49.79} \\
\hline \multicolumn{3}{|c|}{ In the last two weeks, was there any day when you did not take your high blood pressure medication? } & 43.78 & \multicolumn{2}{|c|}{56.22} \\
\hline \multicolumn{3}{|c|}{$\begin{array}{l}\text { Have you ever stopped taking your medications or decreased the dose without first warning your doctor } \\
\text { because you felt worse when you took them? }\end{array}$} & 22.32 & \multicolumn{2}{|c|}{77.68} \\
\hline \multicolumn{3}{|c|}{ When you travel or leave the house, do you sometimes forget to take your medications? } & 42.49 & \multicolumn{2}{|c|}{57.51} \\
\hline \multicolumn{3}{|c|}{ Did you take your high blood pressure medication yesterday? } & 73.39 & \multicolumn{2}{|c|}{26.61} \\
\hline \multicolumn{3}{|c|}{ When you feel your blood pressure is controlled, do you sometimes stop taking your medications? } & 50.21 & \multicolumn{2}{|c|}{49.79} \\
\hline \multicolumn{3}{|c|}{ Have you ever felt distressed for strictly following your high blood pressure treatment? } & 24.46 & \multicolumn{2}{|c|}{75.54} \\
\hline Item 8 & Never & Rarely & Sometimes & Often & Always \\
\hline $\begin{array}{l}\text { 8. How often do you have difficulty in remembering taking all your blood pressure } \\
\text { medications? }\end{array}$ & 75.97 & 9.87 & 12.02 & 1.72 & 0.43 \\
\hline
\end{tabular}

MMAS, Morisky Medication Adherence Scale

Use of the MMAS is protected by US copyright laws. Permission for use is required. A license agreement is available from Donald E. Morisky, ScD, ScM, MSPH, Professor, Department of Community Health Sciences, UCLA Fielding School of Public Health, 650 Charles E. Young Drive South, Los Angeles, CA 90095-1772.

WHO states that adherence is the extent to which the patient follows medical instruction. Adherence also encompasses the numerous healthrelated behaviours (diet and/or executing lifestyle changes) that extend beyond taking prescribed pharmaceutical with recommendations from a health care provider. It estimated that adherence rates range from $50 \%$ to $70 \%$, although the relevant studies varied with respect to study population, duration of follow up, and method used to assess adherence [3]. Morisky et al. classified adherence into 3 categories: low, moderate/medium and high adherence. Patients with high and medium adherence were considered adherent. Fig. 1 shown the adherence level in each primary health care. The mean percentages of low adherence patient were the highest $(57.93 \pm 11.17)$. In contrast, the adherence in all primary health care lower than $50 \%$ that consists of moderate adherence (30.25 \pm 8.03$)$ and high adherence (11.82 \pm 8.18$)$.

According to literature, poor adherence affects the clinical outcome and quality of life, causing risk factor Cardiovascular Diseases (CVD), adverse outcomes such as increased morbidity, mortality and healthcare costs. Although effective medical therapy and evidence-based treatment guidelines for hypertension are readily available, low adherence to antihypertensive medications is prevalent, and blood pressure control is still not optimal, particularly among older populations [9].

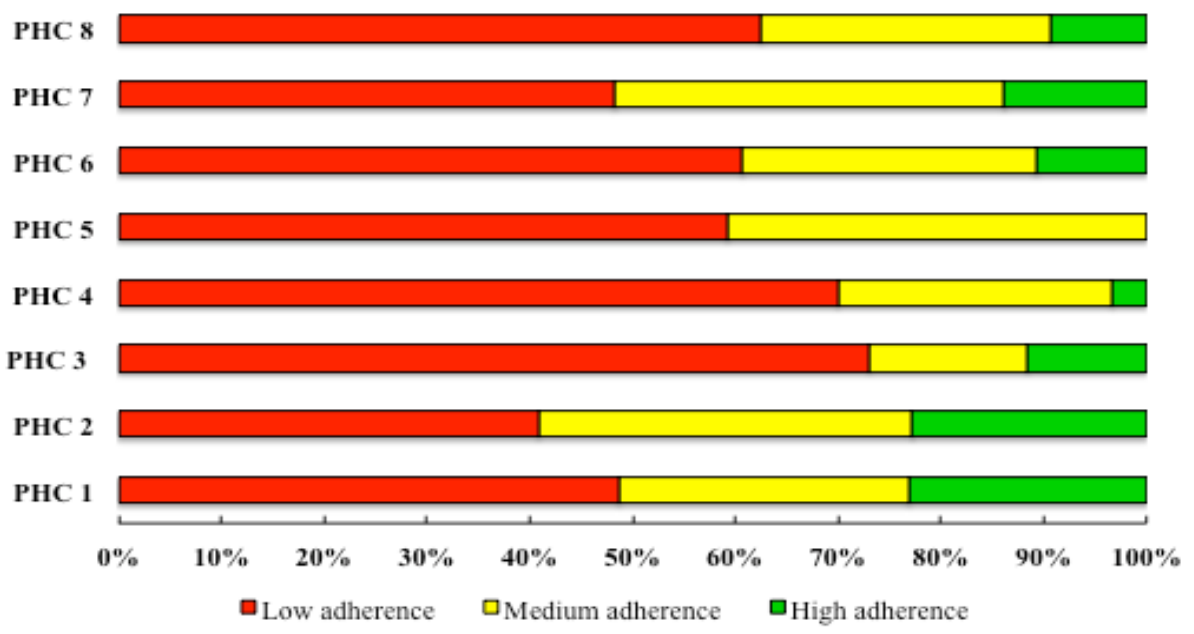

Fig. 1: Adherence level of hypertension treatment in primary health care (PHC) 
Sociodemographic characteristics and life habits of hypertensive patients in every adherence level are shown in table 2. Minimum age of the patients was $32 \mathrm{y}$ and the majority were $60-69 \mathrm{y}(42.6 \%)$ with $64 \%$ of them were females. Only $46.3 \%$ patients practiced some regular physical activity. Smoking was reported by $15.45 \%$ of patients. Antihypertensive monotherapy were prescribed to $79.40 \%$ of patients, such as amlodipine, captopril, and HCT while $29.60 \%$ patients using combination amlodipine and hydrochlorothiazide.

Table 2: Sociodemographic and life habits of hypertensive outpatients

\begin{tabular}{|c|c|c|c|c|c|c|}
\hline \multirow[t]{2}{*}{ Variable } & \multicolumn{2}{|c|}{ Low adherence } & \multicolumn{2}{|c|}{ Medium adherence } & \multicolumn{2}{|c|}{ High adherence } \\
\hline & n & $(\%)$ & $\mathbf{n}$ & $(\%)$ & $\mathbf{n}$ & (\%) \\
\hline \multicolumn{7}{|l|}{ Age } \\
\hline $30-39$ & 2 & 100 & & & & \\
\hline $40-49$ & 14 & 53.85 & 9 & 34.62 & 3 & 11.54 \\
\hline $50-59$ & 36 & 69.23 & 11 & 21.15 & 5 & 9.62 \\
\hline $60-69$ & 54 & 55.10 & 37 & 37.76 & 7 & 7.14 \\
\hline 70 or older & 37 & 67.27 & 12 & 21.82 & 6 & 10.91 \\
\hline \multicolumn{7}{|l|}{ Gender } \\
\hline Female & 90 & 60 & 42 & 28 & 18 & 12 \\
\hline Male & 50 & 60.24 & 28 & 33.73 & 5 & 6.02 \\
\hline \multicolumn{7}{|c|}{ Antihypertensive drugs } \\
\hline Monotherapy & 107 & 57.84 & 61 & 32.97 & 17 & 9.19 \\
\hline Double therapy & 33 & 68.75 & 9 & 18.75 & 6 & 12.50 \\
\hline \multicolumn{7}{|l|}{ Level of schooling } \\
\hline Illiterate & 41 & 100 & & & & \\
\hline Elementary school & 21 & 32.31 & 33 & 50.77 & 11 & 16.92 \\
\hline Junior High school & 32 & 68.09 & 14 & 29.79 & 1 & 2.13 \\
\hline High school & 27 & 52.94 & 16 & 31.37 & 8 & 15.69 \\
\hline College/University & 19 & 65.52 & 7 & 24.14 & 3 & 10.34 \\
\hline \multicolumn{7}{|l|}{ Physical Activity } \\
\hline Yes & 62 & 57.41 & 36 & 33.33 & 10 & 9.26 \\
\hline No & 68 & 54.40 & 34 & 27.20 & 23 & 18.40 \\
\hline \multicolumn{7}{|l|}{ Smoker } \\
\hline Yes & 16 & 44.44 & 12 & 33.33 & 8 & 22.22 \\
\hline No & 114 & 57.87 & 63 & 31.98 & 20 & 10.15 \\
\hline
\end{tabular}

Among patients with lower adherence $76.43 \%$ (45.92\% of total patients) using monotherapy. According to two major studies reviewing therapeutic adherence, one of which covers fifty years of research on the topic, adherence has no apparent association with the demographic characteristics-such as gender, age, socioeconomic status and ethnic group and disease severity [10, 11]. Some predictors, however, have been consistently associated with poor adherence, among which we highlight the complexity of the regimen, the treatment of asymptomatic disease, the presence of psychological problems like depression and medication side effects $[12,13]$.

\section{Limitation of the Study}

We evaluated the treatment adherence of hypertension in primary health care but it didn't correlate with blood pressure target directly, sociodemographic and life habits of the patients. We only described the adherence level of the antihypertensive treatment.

\section{CONCLUSION}

In conclusion, we found that poor adherence was high. The average adherence value according to the MMAS- 8 was $5.2( \pm 1.7)$. Findings from this research we suggest that pharmacist have a great role to support the adherence therapy. Future studies are recommended to confirm our findings, as adherence to medication predicts better outcomes and indicators of poor adherence to a medication regimen are a useful resource for physicians to help identify patients who are most in need of interventions to improve adherence.

We also recommend the implementation of education campaigns to increase awareness about the risk factors, natural history, complications and treatment of hypertension. Global events, such as World Hypertension Day, could be used as a forum to highlight these issues. Patient support groups can be employed to help the nonadherent. Patients who have suffered complications due to nonadherence could be requested to voluntarily share their experiences. Print and audiovisual media would be very helpful in the dissemination of information. Most importantly, though, physicians have to pay special attention to patient education and counselling.

\section{CONFLICT OF INTERESTS}

No potential conflict of interest relevant to this article was reported

\section{REFERENCES}

1. V Chobanian, GL Bakris, HR Black. The seventh report of the joint national committee on prevention, detection, evaluation, and treatment of high blood pressure: the JNC 7 report. JAMA 2003;289:2560-72.

2. PM Kearney, M Whelton, K Reynolds, Muntner P, Whelton PK. Global burden of hypertension: analysis of worldwide data Lancet 2005;365:217-23.

3. World Health Organization (WHO). Adherence to long-term therapies: evidence for action. Geneva (Switzerland); 2003.

4. J Mant, RJ McManus. Does it matter whether patients take antihypertensive medication as prescribed? The complex relationship between adherence and blood pressure control. J Hum Hypertens 2006;20:551-3.

5. T Baune, Y Aljeesh, R Bender. Factors of non-compliance with the therapeutic regimen among hypertensive men and women: a case-control study to investigate risk factors of stroke. Eur J Epidemiol 2005;20:411-9.

6. E Morisky, A Ang, M Krousel-Wood. Predictive validity of medication adherence measure in an outpatient setting. J Clin Hypertens (Greenwich) 2008;10:348-54.

7. Voils RH, Hoyle CT, Thorpe ML, Maciejewski WS, Yancy Jr. Improving the measurement of self-reported medication nonadherence. J Clin Epidemiol 2011;64:250-4.

8. M Krousel-Wood, T Islam, L Webber, RN Re, D Morisky, P Muntner. New medication adherence scale versus pharmacy fill rates in seniors with hypertension. Am J Manag Care 2009;15:59-66.

9. G Pittman, Z Tao, W Chen, GD Stettin. Antihypertensive medication adherence and subsequent healthcare utilization and costs. Am J Manag Care 2010;16:568-76.

10. MR Di Matteo. Variations in patients' adherence to medical recommendations: a quantitative review of $50 \mathrm{y}$ of research. Med Care 2004;42:200-9. 
11. Osterberg L, Blaschke T. Adherence to medication. N Engl J Med 2005;353:487-97.

12. M Krousel-Wood, S Thomasa, P Muntner, D Morisky. Medication adherence: a key factor in achieving blood pressure control and good clinical outcomes in hypertensive patients. Curr Opin Cardiol 2004;19:357-62.

13. J George, YT Phun, MJ Bailey, DC Kong, Stewart K. Development and validation of the medication regimen complexity index. Ann Pharmacother 2004;38:1369-76. 